Nervenarzt 2014 $\cdot$ 85:271-272

DOI 10.1007/s00115-013-3871-z

Online publiziert: 20. Februar 2014

c) Springer-Verlag Berlin Heidelberg 2014

\author{
J.L. Müller, 2 \\ ${ }^{1}$ Asklepios Fachklinikum Göttingen, Forensische Psychiatrie, Göttingen \\ ${ }^{2}$ Ludwig-Meyer-Institut für Forensische Psychiatrie und Psychotherapie, \\ Georg-August-Universität Göttingen, Universitätsmedizin Göttingen
}

\title{
Perspektiven forensischer Psychiatrie und Psychotherapie
}

Über 10.000 Patienten werden bundesweit stationär in Kliniken für forensische Psychiatrie und Psychotherapie behandelt, ambulant werden mehrere Tausend bedingt entlassene Patienten nachbetreut. Lockerungsmissbräuche oder Zwischenfälle mit einschlägigen Straftaten sind Raritäten geworden sind. Durch die ambulante Nachbetreuung in den forensischen Institutsambulanzen wird auch nach einer Entlassung ein hohes Maß an Sicherheit gewährleistet. Inzwischen richtet sich der kritische Blick der Öffentlichkeit vermehrt auf die Rechte der Untergebrachten und den hieraus resultierenden $\mathrm{Re}$ formbedarf als auf die zu schützende Öffentlichkeit. Dies ist uneingeschränkt zu begrüßen, wenn es auch die Behandlung psychisch kranker Patienten, die in Folge ihrer Störung straffällig geworden sind, fördert.

In den letzten Jahren hat insbesondere die gutachtliche Beantwortung forensisch-psychiatrischer Fragestellungen deutlich an Profil gewonnen. Von einer interdisziplinären Arbeitsgruppe am Bundesgerichtshof sind Mindeststandards zur Begutachtung von Schuldfähigkeit und Prognose umgesetzt und verankert worden. Auch hinsichtlich der Therapie werden jetzt entsprechende Standards eingefordert. Diese Behandlungsstandards alsbald umzusetzen, ist eine der ganz wesentlichen Herausforderungen für die forensische Psychiatrie. Bislang zu Unrecht im Schatten der strafrechtlich ausgerichteten forensischen Psychiatrie und Psychotherapie gewinnt jetzt auch die Begutachtung auf zivil- und sozialrechtlichem Gebiet zunehmend an Aufmerksamkeit: Psychiatrische Diagnosen stehen an erster Stelle bei den Rehabilitationsmaßnahmen der Rentenversicherungen und bei den zur vorzeitigen Erwerbsunfähigkeit führenden Erkrankungen. Auch bei diesen gesellschaftspolitisch wichtigen Fragestellungen werden transparente und fachlich begründete Standards für die Begutachtung zu Recht eingefordert. Diese müssen in entsprechenden Qualifizierungen verankert, angeboten und erworben werden. Auch wissenschaftlich ist die forensische Psychiatrie auf einem guten Weg. „Psychopathy“ und Pädophilie repräsentieren forensisch-psychiatrische Forschungsgegenstände, die es auch in die High-impact-Journale geschafft haben. Einhergehend mit dieser zunehmenden Bedeutung forensisch-psychiatrischer Arbeit hat auch die öffentliche Aufmerksamkeit an der forensischen Psychiatrie deutlich zugenommen, wie nicht zuletzt die öffentlichkeitswirksame Bearbeitung des Falles Mollath und die Vielzahl für ein breites Publikum geschriebener Bücher über und aus der Forensik bekunden.

Diese Mut machenden Entwicklungen rechtfertigen durchaus einen Sonderband des Nervenarztes zur forensischen Psychiatrie. Die Inhalte dieses Sonderbandes sollen nun den Blick über den bereits erreichten Wissensstand, der in den Lehrbüchern, Sonderheften und aktuellen Artikeln bereits zugänglich geworden ist, öffnen, sollen aktuelle Entwicklungen erfassen und Perspektiven ausloten. Dies ist das Ziel der Beiträge dieses Sonderheftes Forensische Psychiatrie.

Seilagh Hodgins und Rüdiger MüllerIsberner untersuchen in ihrem Artikel die Evidenz zum Zusammenhang von Schizophrenie und Gewalttätigkeit. Dieses Thema ist von hoher Relevanz sowohl für die Gefährlichkeitseinschätzung und die Risikobeurteilung der betroffenen Patienten als auch um der Stigmatisierung psychischer Krankheiten fundiert entgegenwirken zu können. Die Autoren zeigen auch Möglichkeiten der Intervention und Prävention auf. Ihr Fazit, Kenntnis der Fakten ist die geeigneteste Antistigmatisierung, spornt an.

\section{》) Kenntnis der Fakten ist die geeigneteste Antistigmatisierung}

Harald Dreßing und Klaus Förster greifen mit der Begutachtung der posttraumatischen Belastungsstörungen ein ebenso wichtiges wie umstrittenes Thema auf. Diese Störungsbilder haben in den zurückliegenden Jahren deutlich an Bedeutung bei der Begutachtung gewonnen. In den aktuellen Klassifikations- 
manualen werden die diagnostischen Kriterien modifiziert und unterschiedlich gewichtet, die Ergebnisse der Begutachtung gelten als wenig reliabel und vom einzelnen Gutachter abhängig. Umso wichtiger ist ein Beitrag, der die Standards der Begutachtung zur posttraumatischen Belastungsstörung auf dem aktuellen wissenschaftlichen Kenntnisstand erfasst und abbildet.

Hedwig Eisenbarth nimmt sich des Themas Psychopathy unter einem Genderaspekt an. Längst hat es das Konstrukt Psychopathy auf die Bestsellerlisten populärwissenschaftlicher Bücher geschafft. Man spricht von „white collar psychopathy“ und Gemeindepsychopath und findet psychopathische Züge nahezu ubiquitär. Frau Eisenbarth beschäftigt sich mit der Relevanz dieses Konstrukts bei Frauen, und zwar sowohl bei strafrechtlich auffälligen wie nicht strafrechtlich in Erscheinung getretenen Probandinnen. Ungeachtet aller noch zu leistenden Forschungsanstrengungen erkennt sie neben Gemeinsamkeiten doch geschlechtsbedingte Unterschiede.

Den Stellenwert einer neuen Methodik bei der Begutachtung und Behandlung psychisch kranker Straftäter loten Peter Fromberger, Kirsten Jordan und Jürgen Müller am Beispiel der virtuellen Realität aus. Bei allgemein psychiatrischen Fragestellungen bereits eingesetzt, steht die Anwendung virtueller Techniken für forensisch psychiatrische Fragestellungen noch aus, obwohl das Verfahren großes Potenzial für eine Optimierung der Begutachtung und insbesondere für eine sichere, weil intramural stattfindende Behandlung und Erprobung besitzt. Die Autoren diskutieren allerdings auch Grenzen und mögliche Gefahren bei der Verwendung einer solchen Technik.

Peer Briken und Jürgen Müller beschäftigen sich mit der Frage, inwiefern aktuelle sexualmedizinische Konzepte und operationalisierte Vorgehensweisen von Nutzen bei der Schuldfähigkeitsbegutachtung von Sexualstraftätern sein können. Die Autoren werfen die Frage auf, ob spezifische Items und Definitionen aus aktuellen Prognoseskalen und eigenen Operationalisierungen für die Schuldfähigkeitsbegutachtung von Paraphilien herangezogen werden können.
Mit diesen meines Erachtens zukunftsweisenden Beiträgen des Sonderheftes Forensische Psychiatrie soll noch mehr Interesse geweckt werden an der therapeutischen, der gutachterlichen und auch der wissenschaftlichen Auseinandersetzung mit der forensischen Psychiatrie und Psychotherapie.

\section{Mit herzlichen Grüßen}

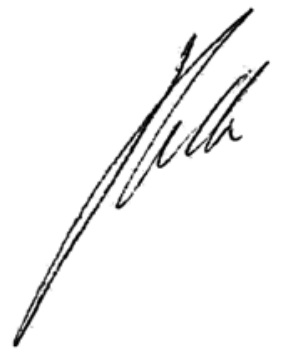

J.L. Müller

\section{Korrespondenzadresse}

Prof. Dr. J.L. Müller

Asklepios Fachklinikum Göttingen,

Forensische Psychiatrie

Rosdorfer Weg 70, 37081 Göttingen

ju.mueller@asklepios.com

Interessenkonflikt. J. L. Müller hat Beraterhonorare der Firma Dr. Pfleger GmbH Bamberg erhalten.

\section{Verordnung von Antipsycho- tika bei Kindern und Jugend- lichen steigt}

Immer mehr Kinder und Jugendliche erhalten Antipsychotika. Zu diesem Ergebnis kamen Wissenschaftler der Universität Marburg, Universität Frankfurt und Universität Bremen bei der Auswertung von Daten der Barmer GEK zur Antipsychotika-Verordnung im Zeitraum von 2005-2012. Insgesamt stieg der Anteil der Verordnungen von $0,23 \%$ auf $0,32 \%$. Dabei war der Zuwachs am deutlichsten bei der Altersgruppe der 10- bis 14- Jährigen und der 15- bis 19-Jährigen. Insbesondere nahmen die Verordnungen atypischer Antipsychotika zu. Die am häufigsten verordneten Substanzen waren Risperidon, Pipamperon, Quetiapin und Tiaprid. Verglichen mit den USA oder Kanada liegen die Zahlen für Deutschland niedrig, im europäischen Vergleich bewegt sich die deutsche Verschreibungspraxis im Mittelfeld.

Nur wenige antipsychotische Substanzen sind für das Kinder- und Jugendalter zugelassen, so dass ihre Verordnung häufig außerhalb der Zulassung (off-label) erfolgt. Generell können durch die Einnahme von Anti-Psychotika Nebenwirkungen entstehen wie z.B. Akathisie, Gewichtszunahme oder Hyperlipidämie. Deshalb ist eine genaue Abwägung und Indikationsstellung durch Kinder- und Jugendpsychiater oder andere Experten für Verhaltensstörungen bei Kindern und Jugendlichen wichtig. Literatur: Deutsches Ärzteblatt (Dtsch Arztebl Int 2014; 111(3): 25 - 40)

Quelle:Deutsches Ärzteblatt, www.aerzteblatt.de 